\title{
Effect of Orthopedic Treatment for Class III Malocclusion on Upper Airways: A Systematic Review and Meta-Analysis
}

\author{
Golnar Havakeshian, Vasiliki Koretsi ${ }^{\circledR}$, Theodore Eliades ${ }^{\circledR}$ and Spyridon N. Papageorgiou * \\ Clinic of Orthodontics and Pediatric Dentistry, Center of Dental Medicine, University of Zurich, \\ 8032 Zurich, Switzerland; g.havakeshian@hotmail.com (G.H.); koretsi.vasiliki@gmail.com (V.K.); \\ theodore.eliades@zzm.uzh.ch (T.E.) \\ * Correspondence: snpapage@gmail.com
}

Received: 18 August 2020; Accepted: 14 September 2020; Published: 18 September 2020

\begin{abstract}
The aim of this systematic review is to compare the effect on the upper airways of orthopedic treatment for skeletal Class III malocclusion with untreated controls. Nine databases were searched up to August 2020 for randomized or nonrandomized clinical trials comparing orthopedic Class III treatment (facemask or chin-cup) to untreated Class III patients. After duplicate study selection, data extraction, and risk of bias assessment (Risk Of Bias In Non-randomized Studies-of Interventions [ROBINS-I]), random-effects meta-analyses of Mean Differences (MDs)/Standardized Mean Differences (SMD) and 95\% Confidence Intervals (CIs) were performed, followed by the Grading of Recommendations Assessment, Development and Evaluation assessment evidence-quality. A total of 10 papers ( 9 unique nonrandomized studies) with 466 patients ( $42.7 \%$ male; average age 9.1 years) were finally included. Limited evidence indicated that compared to normal growth, maxillary protraction with facemask was associated with increases in total airway area $\left(n=1 ; \mathrm{MD}=222.9 \mathrm{~mm}^{2}\right.$; $\left.95 \% \mathrm{CI}=14.0-431.7 \mathrm{~mm}^{2}\right)$, total nasopharyngeal area $(n=4 ; \mathrm{SMD}=1.6 ; 95 \% \mathrm{CI}=1.2-2.0)$, and individual airway dimensions (upper-airway $\mathrm{MD}=2.5 \mathrm{~mm}$; lower-airway $\mathrm{MD}=2.1 \mathrm{~mm}$; upper-pharynx $\mathrm{MD}=1.6 \mathrm{~mm}$; lower-pharynx $\mathrm{MD}=1.0 \mathrm{~mm}$; all $n=6$ ). Subgroup/meta-regression analyses did not find any significant effect-modifiers, while the results were retained 2-5 years postretention. Our confidence in these estimates was, however, very low, due to the inclusion of nonrandomized studies with methodological issues. Limited data from 2 chin-cup studies indicated smaller benefits on airway dimensions. Existing evidence from controlled clinical studies on humans indicates that maxillary protraction for skeletal Class III treatment might be associated with increased airway dimensions, which are, however, mostly minor in magnitude.
\end{abstract}

Keywords: class III malocclusion; maxillary retrognathism; orthopedic treatment; dentofacial orthopedics; orthodontics; clinical trials; systematic review; meta-analysis

\section{Introduction}

\subsection{Background}

Skeletal Class III is multifactorial entity consisting of maxillary retroganthism, mandibular prognathism, or a combination thereof. Its treatment often poses a challenge for the orthodontist. Severe skeletal Class III might considerably affect many aspects of the patient's life, including, among others, psychosocial status, esthetics, mastication, speech and even breathing. The latter might be associated with significant differences in the morphological characteristics of the oropharyngeal airway of patients with Class III deformity. Data from Cone Beam Computerized Tomography (CBCT) indicate that Class III adults might have greater total oropharyngeal volume, but also greater constriction 
areas in the retroglossal and retropalatal compartment of the oropharynx [1]. The most constricted area of the oropharyngeal airway is usually located at the base of the tongue [2]. At the same time, a positive correlation has been reported among tongue volume, pharyngeal airway and Sella-Nasion-B point (SNB) angle, while a negative correlation has been reported between tongue volume and A point-Nasion-B point (ANB) angle [3]. Finally, Class III patients might have higher risk of being mouth breathers than Class I patients (32\% and 23\%, respectively) [4].

From the available Class III treatment options, orthopedic modification as well as orthognathic surgery might be expected to exert the greatest influence on the anatomy and volume of the airway. Specifically, significantly more orthognathic patients report a perceived improvement in breathing through maxillary advancement (with or without mandibular setback) $(95 \%)$ than with mandibular setback alone (56\%) [5]. Bimaxillary surgery results in greater increases than mandibular setback in the volume of the airway, which are primarily apparent due to an increase in the retropalatal region [6]. For growing Class III patients, orthopedic appliances are sometimes used to protract the maxilla, restrict or redirect mandibular growth, or both, while at the same time, both approaches lead to a mandibular rotation. However, consensus regarding the impact that these therapeutic approaches might have on the airway has not been reached in the literature. Some studies have reported a favorable effect on the dimensions of the airway [7-10], while others have not confirmed such an effect [11,12]. A recent systematic review on the topic [13] reported that maxillary protraction can increase postpalatal and nasopharyngeal airway dimensions in growing skeletal class III subjects with maxillary retrusion. However, that review included studies published only up to 2017, and its conclusions might be influenced by many existing issues like the lack of an a priori protocol [14], incomplete handling of risk of bias within studies according to the latest Cochrane guidelines [15], issues with the data synthesis (improper model selection, double-counting of multiple arms from single studies and a lack of sensitivity analyses) [16], and the lack of a formal assessment of the review's quality of evidence [17]. Finally, that review only assessed the impact of maxillary protraction on airway dimensions and did not include mandibular restriction with chin-cup.

\subsection{Objective}

The aim of this systematic review was to assess the effect of different orthopedic appliances on the upper airway dimension in growing patients with class III malocclusion. The research question was "can orthopedic treatment of growing Class III patients with facemask or chin-cup modify the upper airway dimensions to a degree greater than what can be attributed to normal growth?".

\section{Materials and Methods}

\subsection{Protocol and Registration}

A review protocol was designed and registered a priori (PROSPERO CRD42020148142), while post hoc changes were transparently reported (Supplementary Materials S1). The conduct and reporting of this review is based on the Cochrane Handbook [18] and PRISMA statement [19], respectively.

\subsection{Eligibility Criteria}

Clinical studies on human pre-adolescent and adolescent patients of any sex or ethnicity with skeletal Class III malocclusion as an indication for orthopedic treatment were included (Supplementary Materials S2). No limitations concerning language, publication year, or status were applied. The primary outcome of this review was total dimensions of the upper airways. Secondary outcomes included other dimensions of the separate airway compartments in linear or areal measurements. 


\subsection{Information Sources and Search}

Nine electronic databases were systematically searched without any restrictions for publication date, type, and language from inception up to 4 August 2020 (Supplementary Materials S3), while Directory of Open Access Journals, Digital Dissertations, metaRegister of Controlled Trials, World Health Organization, and Google Scholar, as well as the reference lists of eligible articles or existing systematic reviews were manually searched for any additions.

\subsection{Study Selection}

Two authors (G.H., V.K.) screened the titles and/or abstracts of studies retrieved from the searches to identify articles that potentially met the inclusion criteria, before moving to their full-texts. Any differences between the two reviewers were resolved by discussion with a third author (S.N.P.).

\subsection{Data Collection Process and Items}

Data collection from the identified reports was conducted using predefined and piloted forms covering: (a) study characteristics (design, clinical setting, country), (b) patient characteristics (age, sex), (c) inclusion criteria/malocclusion characteristics, (d) treatment characteristics (appliance and duration), (e) post-treatment follow-up duration, and (f) outcome assessment. Data were extracted by two authors (G.H., V.K.) with the aforementioned way to resolve discrepancies.

\subsection{Risk of Bias of Individual Studies}

The risk of bias of included nonrandomized studies was assessed with the ROBINS-I ("Risk Of Bias In Nonrandomized Studies-of Interventions") [15]. Assessment of the risk of bias within individual studies was likewise independently performed by two authors (G.H., V.K.) with the same approach being applied to resolve discrepancies.

\subsection{Data Synthesis and Summary Measures}

An effort was made to include all existing trials in the analysis; where data were missing, they were calculated by us (Supplementary Materials S1). As duration of orthopedic effects on the airways might be affected by patient anatomy or growth potential, appliance characteristics and patient compliance, a random-effects model was deemed appropriate to calculate the average distribution of true effects [20] with a restricted maximum likelihood variance estimator [21], using Mean differences (MDs) or Standardized Mean Differences (SMDs) and their corresponding 95\% confidence intervals (CIs). The produced forest plots were augmented with contours denoting the magnitude of the observed effects to assess heterogeneity, clinical relevance and imprecision [16] (Supplementary Materials S1).

The extent and impact of between-study heterogeneity was gauged visually and quantified with $\operatorname{tau}^{2}$ (absolute heterogeneity) and the $\mathrm{I}^{2}$ statistic (relative heterogeneity; inconsistency). Inconsistency over $75 \%$ was arbitrarily considered as high, but we also considered where the inconsistency was localized on the forest plot and our uncertainty around these estimates [22]. Ninety-five per cent predictive intervals, integral in the correct interpretation of random-effects meta-analyses [23], were estimated for meta-analyses of $\geq 3$ trials to incorporate observed heterogeneity and provide a range of possible effects for a future clinical setting.

\subsection{Additional Analyses and Risk of Bias across Studies}

Many subgroup and meta-regression analyses were originally planned (Supplementary Materials S1), but only some could be performed in the end. Likewise, reporting biases were planned but could not be assessed in this review.

The overall quality of meta-evidence (i.e., the strength of clinical recommendations) was rated using the Grades of Recommendations, Assessment, Development, and Evaluation (GRADE) approach [17] 
following recent guidance on synthesizing nonrandomized studies [24] and summary of findings tables were constructed using an improved format [25] (Supplementary Materials S1).

Robustness of the results was planned to be checked a priori with sensitivity analyses based on (a) baseline similarity of treated-control group in airway measurements, and (b) sample size, while some sensitivity analyses could not be performed (Supplementary Materials S1).

All the analyses were run in Stata version 14.0 (StataCorp LP, College Station, TX, USA) by one author (SNP) with an openly provided dataset [26]. All $p$ values were two-sided with $\alpha=5 \%$, except for the test of between-studies or between-subgroups heterogeneity, where $\alpha$-value was set at $10 \%$ [27].

\section{Results}

\subsection{Study Selection}

The electronic literature search yielded 849 results, while one study was manually identified (Figure 1). After duplicate removal and screening of titles/abstracts against the predefined eligibility criteria (Supplementary Materials S4), the full texts of 104 papers were checked. Eventually, 10 papers pertaining to 9 unique studies (1 prospective and 9 retrospective nonrandomized studies; Table 1), which were published as journal papers, were finally included [8,11,12,28-34].

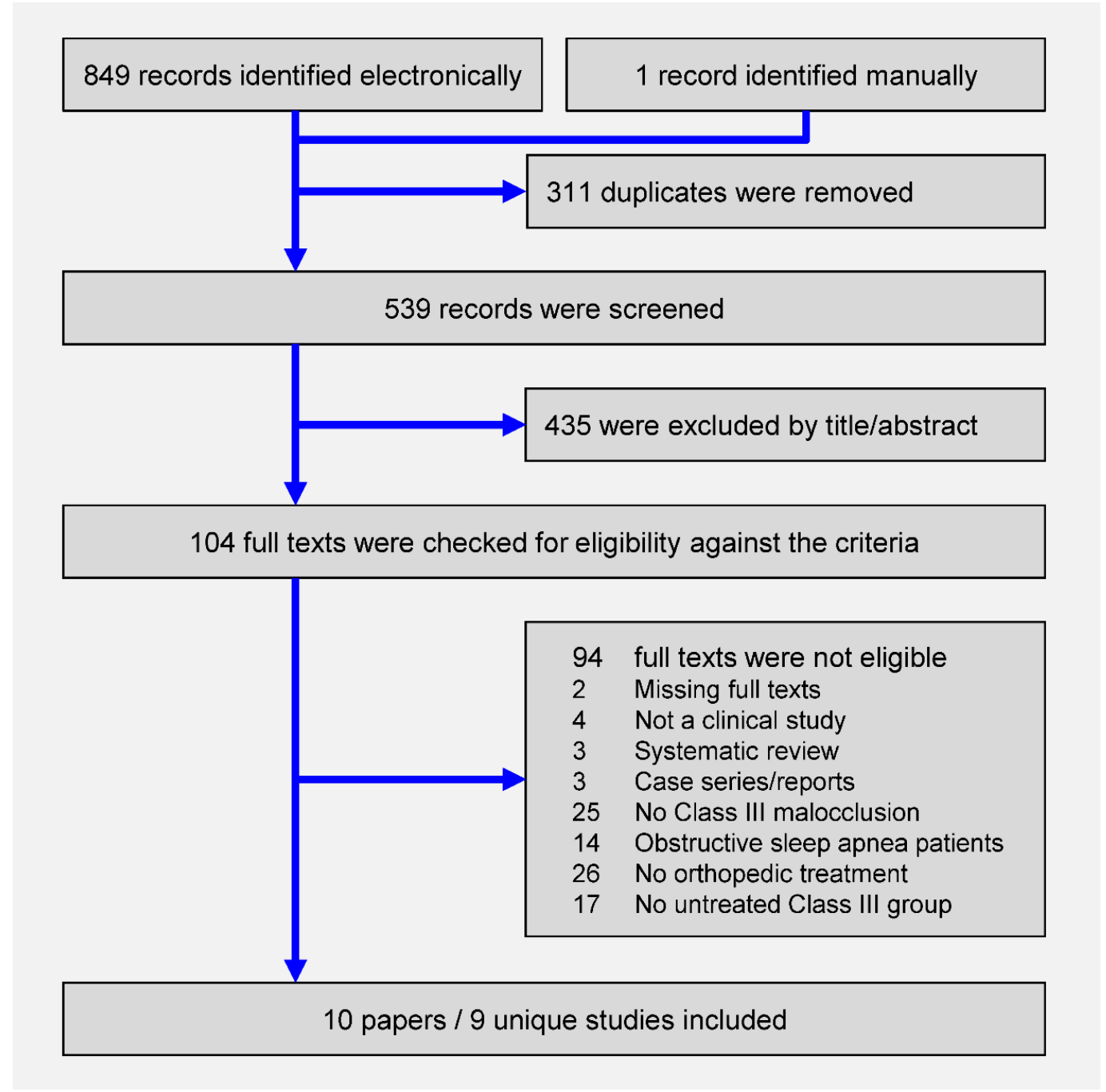

Figure 1. Preferred Reporting Items for Systematic Reviews and Meta-Analyses flow diagram for the identification and selection of studies. 
Table 1. Characteristics of the included studies.

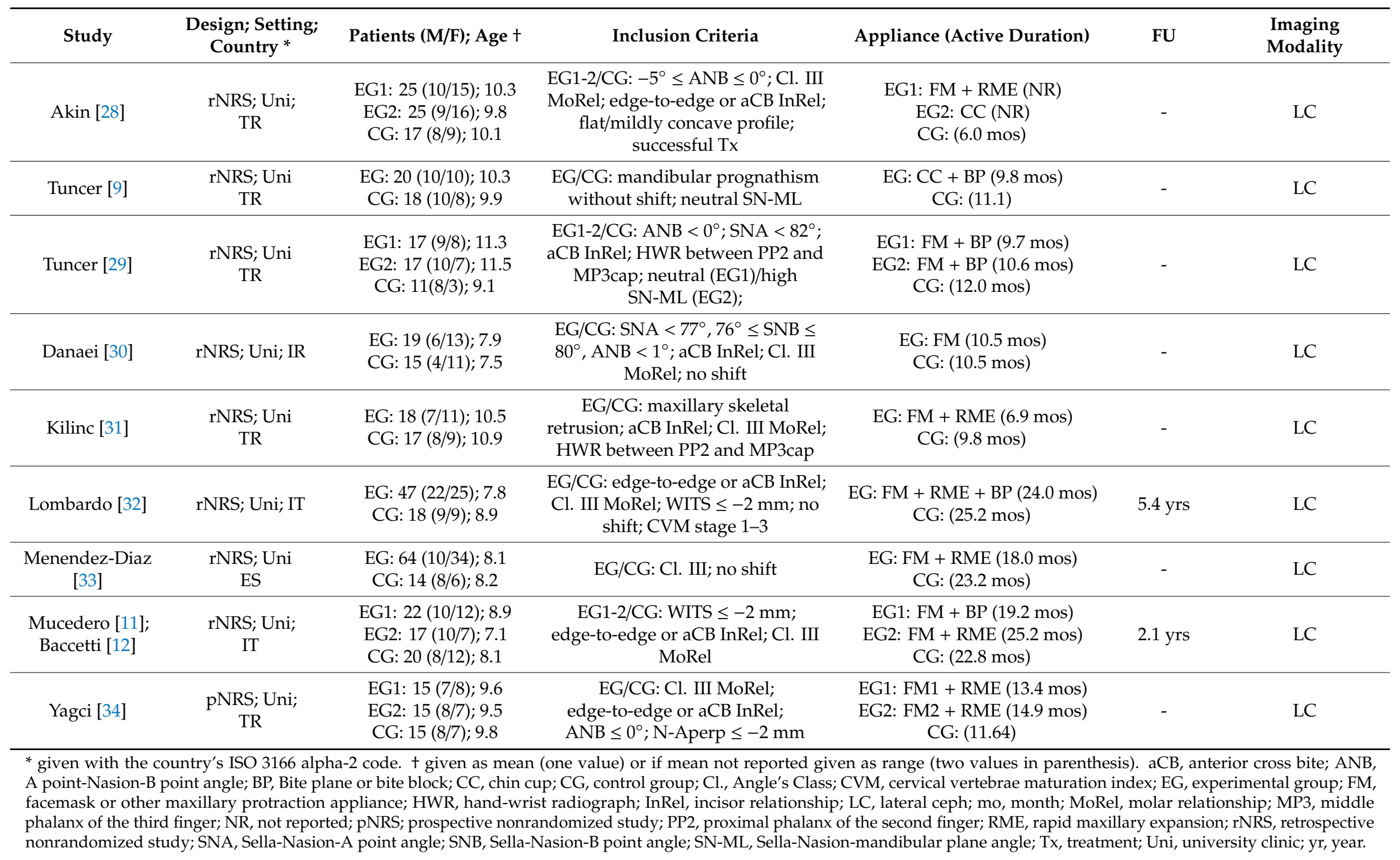




\subsection{Study Characteristics}

All primary studies were conducted in university clinics $(n=9 ; 100 \%)$ and originated from four different countries (Iran, Italy, Spain, Turkey) (Table 1). A total of 321 treated and 145 untreated Class III patients were included, with a median total sample of 45 patients per included study (range 34 to 78 patients per study). All studies reported patient sex and age, with 199 of 466 patients (42.7\%) being male and the mean patient age being 9.1 years.

Seven of the included studies assessed maxillary protraction with facemask/reverse headgear, one assessed mandibular restraint with chin-cup, and one included one facemask arm and one chin-cup arm. Among the eight facemask studies, four incorporated maxillary expansion, two included a bite-plane (or bite-blocks) and one included both maxillary expansion and bite-plane. Average treatment duration was 15.1 months for facemask treatment (7 studies; range 6.9 to 24.0 months) and 9.8 months for chin-cup ( 1 study), while the average observation period for the untreated controls was 14.7 months (9 studies; 6.0 to 25.2 months). All studies measured outcomes before and directly after treatment, while two studies additional remeasured outcomes postretention after an additional average period of 2.1 to 5.1 years. Finally, all studies assessed the effects of treatment on airways with lateral cephalograms.

\subsection{Risk of Bias within Studies}

The included nonrandomized trials presented several issues influencing their risk for bias (Table 2). All studies except one $(n=8 ; 89 \%)$ were retrospective, and patient selection could influence the results of treatment for most of them $(n=8 ; 89 \%)$. For at least three studies $(33 \%)$, treated/control patients were followed/observed for different durations, while baseline differences in age, sex, malocclusion severity or airway measurements existed for 1-3 studies (11-33\%). No study performed blinded outcome measurement, while for at least four studies $(44 \%)$, the treated and control populations originated from different sources. All included studies were judged to be in critical risk of bias, as issues existed for at least three domains per study. 
Table 2. Detailed assessment of included nonrandomized studies with the ROBINS-I tool.

\begin{tabular}{|c|c|c|c|c|c|c|c|c|c|}
\hline Reference & $\begin{array}{c}\text { Akin } \\
{[28]}\end{array}$ & $\begin{array}{c}\text { Tuncer } \\
{[9]}\end{array}$ & $\begin{array}{c}\text { Tuncer } \\
{[29]}\end{array}$ & $\begin{array}{c}\text { Danaei } \\
{[30]}\end{array}$ & $\begin{array}{c}\text { Kilinc } \\
{[31]}\end{array}$ & $\begin{array}{c}\text { Lombardo } \\
{[32]}\end{array}$ & $\begin{array}{l}\text { Menendez } \\
\text {-Diaz [33] }\end{array}$ & $\begin{array}{l}\text { Mucedero [11]; } \\
\text { Baccetti [12] }\end{array}$ & $\begin{array}{c}\text { Yagci } \\
{[34]}\end{array}$ \\
\hline Was the study prospective? & $\mathrm{N}$ & $\mathrm{N}$ & $\mathrm{N}$ & $\mathrm{N}$ & $\mathrm{N}$ & $\mathrm{N}$ & $\mathrm{N}$ & $\mathrm{N}$ & $\mathrm{Y}$ \\
\hline $\begin{array}{l}\text { Was selection of patients based on any factor that } \\
\text { could influence airways post treatment (age, sex, } \\
\text { skeletal configuration, compliance, breakages)? }\end{array}$ & $\mathrm{Y}$ & PY & Y & $\mathrm{Y}$ & PY & $\mathrm{PN}$ & PY & PY & $\mathrm{Y}$ \\
\hline Were treated/untreated groups clearly defined? & $\mathrm{Y}$ & $\mathrm{Y}$ & Y & $\mathrm{Y}$ & $\mathrm{Y}$ & $\mathrm{Y}$ & $\mathrm{Y}$ & $\mathrm{Y}$ & $\mathrm{Y}$ \\
\hline $\begin{array}{l}\text { Was the observation period similar for } \\
\text { treated/untreated patients? }\end{array}$ & NI & $\mathrm{Y}$ & $\mathrm{PN}$ & PY & $\mathrm{PN}$ & $\mathrm{Y}$ & $\mathrm{PN}$ & $\mathrm{PN}$ & PY \\
\hline $\begin{array}{l}\text { Were treated/untreated patients similar in terms of } \\
\text { baseline age? }\end{array}$ & $\mathrm{Y}$ & PY & PN & $\mathrm{Y}$ & $\mathrm{Y}$ & PY & $Y$ & PY & $Y$ \\
\hline $\begin{array}{l}\text { Were treated/untreated patients similar in terms of } \\
\text { baseline sex? }\end{array}$ & Y & PY & PN & Y & $\mathrm{N}$ & PY & $\mathrm{N}$ & $\mathrm{N}$ & $\mathrm{Y}$ \\
\hline $\begin{array}{l}\text { Were treated/untreated patients similar in terms of } \\
\text { dental/skeletal malocclusion? }\end{array}$ & $\mathrm{Y}$ & PY & $\mathrm{N}$ & $\mathrm{NI}$ & $Y$ & PY & $Y$ & $Y$ & $Y$ \\
\hline $\begin{array}{c}\text { Were treated/untreated patients similar in terms of } \\
\text { baseline airways? }\end{array}$ & PN & $\mathrm{PN}$ & Y & $Y$ & $Y$ & PY & $Y$ & $\mathrm{Y}$ & $\mathrm{N}$ \\
\hline $\begin{array}{l}\text { Was the use of any other appliances/adjuncts the } \\
\text { same among treated/untreated patients? }\end{array}$ & NA & NA & NI & NI & NA & NA & $\mathrm{NI}$ & NA & NA \\
\hline $\begin{array}{l}\text { Was outcome measurement similar for } \\
\text { treated/untreated patients? }\end{array}$ & $\mathrm{Y}$ & $Y$ & $Y$ & $Y$ & $Y$ & Y & $Y$ & $Y$ & $Y$ \\
\hline $\begin{array}{l}\text { Was outcome measurement done blindly for both } \\
\text { treated/untreated patients? }\end{array}$ & $\mathrm{N}$ & $\mathrm{N}$ & $\mathrm{N}$ & $\mathrm{N}$ & $\mathrm{N}$ & $\mathrm{N}$ & $\mathrm{N}$ & $\mathrm{N}$ & $\mathrm{N}$ \\
\hline $\begin{array}{c}\text { Were treated/untreated patients treated/observed at } \\
\text { the same place/time? }\end{array}$ & NI & NI & $\mathrm{N}$ & NI & $\mathrm{N}$ & $\mathrm{N}$ & $\mathrm{N}$ & $\mathrm{N}$ & $Y$ \\
\hline
\end{tabular}

N, no; NA, not applicable; NI, no information; PN, probably not; PY, probably yes; $\mathrm{Y}$, yes. 
Table 3. Random-effects meta-analyses on the effect of facemask (with/without maxillary expansion) on upper airway dimensions.

\begin{tabular}{|c|c|c|c|c|c|c|}
\hline Outcome & $n$ & Effect $(95 \% \mathrm{CI})$ & $p$ & $I^{2}(95 \% C I)$ & $\operatorname{tau}^{2}(95 \% \mathrm{CI})$ & 95\% Prediction \\
\hline \multicolumn{7}{|c|}{ Post-treatment } \\
\hline Total nasopharyngeal area $\left(\mathrm{mm}^{2}\right)$ & 4 & $\mathrm{SMD}=1.62(1.20,2.04)$ & $<0.001$ & $23 \%(0 \%, 91 \%)$ & $0.04(0,1.42)$ & $0.34,2.90$ \\
\hline Adenoidal nasopharyngeal area $\left(\mathrm{mm}^{2}\right)$ & 2 & $\mathrm{MD}=0.34(-0.10,0.77)$ & 0.13 & $0 \%(0 \%, 99 \%)$ & $0(0,7.60)$ & - \\
\hline Aerial nasopharyngeal area $\left(\mathrm{mm}^{2}\right)$ & 2 & $\mathrm{MD}=1.29(0.80,1.77)$ & $<0.001$ & $0 \%(0 \%, 99 \%)$ & $0(0,14.38)$ & - \\
\hline Oropharyngeal area $\left(\mathrm{mm}^{2}\right)$ & 2 & $\mathrm{MD}=-0.18(-1.65,1.29)$ & 0.81 & $89 \%(24 \%, 100 \%)$ & $1.00(0.04,141.23)$ & - \\
\hline Upper adenoid size (AD2-H; mm) & 3 & $\mathrm{MD}=0.59(-0.52,1.70)$ & 0.30 & $57 \%(0 \%, 98 \%)$ & $0.55(0,24.22)$ & $-11.24,12.42$ \\
\hline Lower adenoid size (AD1-Ba; mm) & 3 & $\mathrm{MD}=0.12(-2.20,2.44)$ & 0.92 & $76 \%(0 \%, 99 \%)$ & $3.11(0,76.33)$ & $-26.88,27.12$ \\
\hline Upper airway dimension (PNS-AD2; mm) & 6 & $\mathrm{MD}=2.45(0.97,3.92)$ & 0.001 & $87 \%(64 \%, 97 \%)$ & $2.83(0.74,15.17)$ & $-2.67,7.57$ \\
\hline Lower airway dimension (PNS-AD1; mm) & 6 & $\mathrm{MD}=2.10(1.50,2.70)$ & $<0.001$ & $5 \%(0 \%, 87 \%)$ & $0.04(0,4.83)$ & $1.11,3.09$ \\
\hline McNamara's upper pharynx dimension (mm) & 6 & $\mathrm{MD}=1.59(0.57,2.62)$ & 0.002 & $73 \%(15 \%, 95 \%)$ & $1.08(0.07,7.56)$ & $-1.63,4.82$ \\
\hline McNamara's lower pharynx dimension (mm) & 6 & $\mathrm{MD}=1.02(0.17,1.88)$ & 0.02 & $70 \%(16 \%, 94 \%)$ & $0.69(0.06,4.90)$ & $-1.58,3.63$ \\
\hline \multicolumn{7}{|c|}{ Postretention } \\
\hline Upper adenoid size (AD2-H; mm) & 2 & $\mathrm{MD}=-1.13(-4.25,2.00)$ & 0.48 & $72 \%(0 \%, 100 \%)$ & $3.70(0,641.91)$ & - \\
\hline Lower adenoid size (AD1-Ba; mm) & 2 & $\mathrm{MD}=-2.67(-4.63,-0.70)$ & 0.008 & $14 \%(0 \%, 99 \%)$ & $0.31(0,275.16)$ & - \\
\hline Upper airway dimension (PNS-AD2; mm) & 2 & $\mathrm{MD}=3.71(0.80,6.62)$ & 0.01 & $65 \%(0 \%, 100 \%)$ & $2.91(0,563.83)$ & - \\
\hline Lower airway dimension (PNS-AD1; mm) & 2 & $\mathrm{MD}=3.59(1.75,5.44)$ & $<0.001$ & $0 \%(0 \%, 98 \%)$ & $0(0,132.16)$ & - \\
\hline McNamara's upper pharynx dimension (mm) & 2 & $\mathrm{MD}=2.27(0.80,3.74)$ & 0.003 & $0 \%(0 \%, 98 \%)$ & $0(0,89.15)$ & - \\
\hline McNamara's lower pharynx dimension (mm) & 2 & $\mathrm{MD}=1.84(-2.08,5.75)$ & 0.36 & $85 \%(\mathrm{NC})$ & $6.80(\mathrm{NC})$ & - \\
\hline
\end{tabular}

$\mathrm{CI}$, confidence interval; $\mathrm{MD}$, mean difference; $\mathrm{SMD}$, standardized mean difference. Statistical significance is denoted in bold. 


\subsection{Results of Individual Studies and Data Synthesis}

\subsubsection{Maxillary Protraction with Facemask/Reverse Headgear}

Eight studies provided various measurements of linear distances or area measurements of airways post-treatment or postretention. The results of the performed meta-analyses are given in Table 3, while the results of single studies that could not be pooled in meta-analyses are given in Supplementary Materials S5.

The review's primary outcome of total airway area was assessed by a single study, which found a statistically and clinically relevant increase in airway area post-treatment $\left(\mathrm{MD}=222.86 \mathrm{~mm}^{2}\right.$; $\left.95 \% \mathrm{CI}=14.04-431.68 \mathrm{~mm}^{2} ; p=0.04\right)$. As far as secondary outcomes, statistically significant post-treatment increases were seen for the facemask group compared to the control group regarding total nasopharyngeal area (4 studies; $\mathrm{SMD}=1.62 ; 95 \% \mathrm{CI}=1.20-2.04 ; p<0.001$; $\mathrm{I}^{2}=23 \%$; Supplementary Materials S6), aerial nasopharyngeal area (2 studies; $\mathrm{MD}=1.29 \mathrm{~mm}^{2}$; $95 \% \mathrm{CI}=0.80-1.77 \mathrm{~mm}^{2} ; p<0.001 ; \mathrm{I}^{2}=0 \%$ ), upper airway dimensions (6 studies; $\mathrm{MD}=2.45 \mathrm{~mm}$; 95\% CI = 0.97-3.92 mm; $p=0.001 ; \mathrm{I}^{2}=87 \%$; Supplementary Materials S7), lower airway dimensions (6 studies; $\mathrm{MD}=2.10 \mathrm{~mm} ; 95 \% \mathrm{CI}=1.50-2.70 \mathrm{~mm} ; p<0.001 ; \mathrm{I}^{2}=5 \%$; Supplementary Materials S8), McNamara's upper pharynx dimensions (6 studies; $\mathrm{MD}=1.59 \mathrm{~mm} ; 95 \% \mathrm{CI}=0.57-2.62 \mathrm{~mm} ; p=0.002$; $\mathrm{I}^{2}=73 \%$; Supplementary Materials S9), and McNamara's lower pharynx dimensions (6 studies; $\mathrm{MD}=1.02 \mathrm{~mm} ; 95 \% \mathrm{CI}=0.17-1.88 \mathrm{~mm} ; p=0.02 ; \mathrm{I}^{2}=70 \%$; Supplementary Materials S10). Observed heterogeneity was acceptable in all instances, except for the meta-analysis of upper airway dimensions $\left(\mathrm{I}^{2}=87 \%\right)$, but all studies were on the right side of the forest plot (Supplementary Materials S7), so judgment about the beneficial effects of treatment was not influenced by it, but rather, only the precise quantification of the treatment's effects. The 95\% prediction intervals were inconsistent (included both negative and negative values) for most meta-analyses, with only total pharyngeal area and lower airway dimension being consistent, meaning that we can consistently expect significant benefits in every future scenario.

Apart from these meta-analyses, individual studies reported on several increases in airway dimensions (Supplementary Materials S5) that were either statistically nonsignificant or clinically nonrelevant, apart from a relevant increase in oropharynx dimensions.

Finally, postretention data 2.1 to 5.4 years after treatment were available from two studies (Table 3). Lower adenoid side was significantly lower in treated patients compared to controls ( 2 studies; $\mathrm{MD}=-2.67 \mathrm{~mm} ; 95 \% \mathrm{CI}=-4.63--0.70 \mathrm{~mm} ; p=0.008$ ), a novel finding that was not seen directly post-treatment. The dimensions of the upper and lower airways were still significantly larger among treated than untreated patients and to a greater extent than directly post-treatment (MD of $3.71 \mathrm{~mm}$ versus $2.45 \mathrm{~mm}$ for the upper airway; MD of $3.59 \mathrm{~mm}$ versus $2.10 \mathrm{~mm}$ for the lower airway). Treatment-related benefits in the dimensions of the pharynx were likewise increased postretention, though this increase was statistically significant only for the upper pharynx.

\subsubsection{Mandibular Restraint with Chin-Cup}

Two studies provided various measurement on linear distances or area measurements of airways post-treatment (Supplementary Materials S11), most of which were not significant-with two exceptions. One study reported a post-treatment increase of the nasopharyngeal area $\left(\mathrm{MD}=10,183.0 \mathrm{~mm}^{2} ; 95 \% \mathrm{CI}=10,074.3-10,291.8 \mathrm{~mm}^{2} ; p<0.001\right)$, which was both statistically significant and clinically relevant. The same study reported a post-treatment reduction of the oropharyngeal area $\left(\mathrm{MD}=-8231.0 \mathrm{~mm}^{2} ; 95 \% \mathrm{CI}=-10,616.5--5845.5 \mathrm{~mm}^{2} ; p<0.001\right)$, which was statistically significant but clinically irrelevant.

\subsection{Additional Analyses, Risk of Bias across Studies, and Quality of Evidence}

Several subgroup analyses, meta-regressions and assessments for reporting biases were originally planned in the protocol, but could ultimately not be performed (Supplementary Materials S1). 
Selected subgroup and meta-regression analyses on the post-treatment effects of maxillary protraction (for meta-analyses with $\geq 5$ studies) found no significant influence of patient age, sex, baseline airway dimensions, inclusion of maxillary expansion or treatment duration (Table 4). Likewise, one study found no significant differences between normodivergent or hyperdivergent patients, and another study found no significant differences between two facemask designs.

Table 4. $p$ values from subgroup analyses and meta-regressions.

\begin{tabular}{cccccc}
\hline Outcome & Age & Male\% & Baseline Airway & RME & Tx Duration \\
\hline $\begin{array}{c}\text { Upper airway dimension } \\
\text { (PNS-AD2; mm) }\end{array}$ & 0.45 & 0.65 & 0.69 & 0.29 & 0.27 \\
\hline $\begin{array}{c}\text { Lower airway dimension } \\
\text { (PNS-AD1; mm) }\end{array}$ & 0.98 & 0.15 & 0.37 & 0.65 & 0.26 \\
\hline $\begin{array}{c}\text { McNamara's upper pharynx } \\
\text { dimension (mm) }\end{array}$ & 0.46 & 0.23 & 0.46 & 0.81 & 0.33 \\
\hline $\begin{array}{c}\text { McNamara's lower pharynx } \\
\text { dimension (mm) }\end{array}$ & 0.13 & 0.65 & 0.45 & 0.70 & 0.67 \\
\hline
\end{tabular}

RME, rapid maxillary expansion, $\mathrm{Tx}$, treatment.

The quality of evidence (Table 5) for the main analyses on the post-treatment effects of facemask was, in all cases, very low, due to the inclusion of retrospective nonrandomized studies with critical risk of bias. The quality of evidence about the primary outcome of total airway area was additionally downgraded due to imprecision, as a single study with a limited sample size contributed to this. The GRADE analysis indicates that further research in terms of well-designed studies is very likely to have an important impact which will likely change our current estimates of effect. 
Table 5. Summary of Findings Table according to GRADE approach.

\begin{tabular}{|c|c|c|c|c|}
\hline \multicolumn{5}{|c|}{ Anticipated Absolute Effects (95\% CI) } \\
\hline $\begin{array}{c}\text { Outcome } \\
\text { Studies (Patients) }\end{array}$ & Control (Growth) & Maxillary Protraction & $\begin{array}{l}\text { Quality of the Evidence } \\
\text { (GRADE) } b\end{array}$ & $\begin{array}{l}\text { What Happens with Maxillary } \\
\text { Protraction }\end{array}$ \\
\hline $\begin{array}{l}\text { Total airway area } \\
35 \text { patients (1 study) }\end{array}$ & $-38.4 \mathrm{~mm}^{2}$ & $\begin{array}{c}223 \mathrm{~mm}^{2} \text { greater } \\
\text { (14.0 to } 431.7 \mathrm{~mm}^{2} \text { greater) }\end{array}$ & $\begin{array}{l}\bigcirc 000 \text { very low } \mathrm{c}, \mathrm{d} \\
\text { due to bias, imprecision }\end{array}$ & $\begin{array}{l}\text { Might be associated with } \\
\text { greater airway area }\end{array}$ \\
\hline $\begin{array}{l}\text { Upper airway dimensions } \\
316 \text { patients (6 studies) }\end{array}$ & $0.3 \mathrm{~mm}^{\mathrm{a}}$ & $\begin{array}{l}2.5 \mathrm{~mm} \text { greater } \\
\text { (1.0 to } 3.9 \mathrm{~mm} \text { greater) }\end{array}$ & $\begin{array}{l}0000 \text { very low }{ }^{c} \\
\text { due to bias }\end{array}$ & $\begin{array}{l}\text { Might be associated with greater } \\
\text { upper airway dimensions }\end{array}$ \\
\hline $\begin{array}{l}\text { Lower airway dimensions } \\
316 \text { patients (6 studies) }\end{array}$ & $0.5 \mathrm{~mm}^{\mathrm{a}}$ & $\begin{array}{c}2.1 \mathrm{~mm} \text { greater } \\
\text { (1.5 to } 2.7 \mathrm{~mm} \text { greater) }\end{array}$ & $\begin{array}{l}0000 \text { very low }{ }^{c} \\
\text { due to bias }\end{array}$ & $\begin{array}{l}\text { Might be associated with greater } \\
\text { lower airway dimensions }\end{array}$ \\
\hline $\begin{array}{l}\text { Upper pharynx dimensions (McNamara's) } \\
323 \text { patients (6 studies) }\end{array}$ & $0.6 \mathrm{~mm}^{\mathrm{a}}$ & $\begin{array}{l}1.6 \mathrm{~mm} \text { greater } \\
\text { (0.6 to } 2.6 \mathrm{~mm} \text { greater) }\end{array}$ & $\begin{array}{l}000 \bigcirc \text { very low }^{\mathrm{c}} \\
\text { due to bias }\end{array}$ & $\begin{array}{l}\text { Might be associated with greater } \\
\text { upper pharynx dimensions }\end{array}$ \\
\hline $\begin{array}{l}\text { Lower pharynx dimensions (McNamara's) } \\
\qquad 323 \text { patients (6 studies) }\end{array}$ & $0.1 \mathrm{~mm}^{\mathrm{a}}$ & $\begin{array}{c}1.0 \mathrm{~mm} \text { greater } \\
(0.2 \text { to } 13.9 \mathrm{~mm} \text { greater })\end{array}$ & $\begin{array}{l}\bigcirc 0 \bigcirc \bigcirc \text { very low }^{c} \\
\text { due to bias }\end{array}$ & $\begin{array}{l}\text { Might be associated with greater } \\
\text { lower pharynx dimensions }\end{array}$ \\
\hline
\end{tabular}

Intervention: orthopedic maxillary protraction with facemask or reverse headgear/Population: pre-adolescent children with skeletal Class III malocclusion/Setting: university clinics (Iran, Italy, Spain, Turkey). a Response in the control group is based on random-effects meta-analysis of the control groups of included studies. ${ }^{b}$ Starts from "high". c Downgraded by three levels for bias due to the inclusion of retrospective nonrandomized studies with serious risk of bias. ${ }^{\mathrm{d}}$ Downgraded by one level for imprecision due to the inclusion of an inadequate sample. CI, confidence interval; GRADE, Grading of Recommendations Assessment, Development and Evaluation, $\bigcirc \bigcirc \bigcirc \bigcirc$, downgraded by 4 points. 


\subsection{Sensitivity Analysis}

A sensitivity analysis according to sample size and baseline similarity of treated/control patients (Supplementary Materials S12) indicated relative robustness of the results.

\section{Discussion}

\subsection{Evidence in Context}

The current review summarizes and critically appraises existing evidence from clinical research comparing the effects of Class III orthopedic treatment on airway dimensions to untreated Class III controls. A total of 10 papers (9 studies) including 321 treated and 145 untreated Class III patients were finally identified as eligible and contributed to data synthesis.

Maxillary protraction with a facemask, with or without an expander, was shown to result in statistically significant increases in airway dimensions directly after treatment compared to what could be expected by Class III growth alone. Specifically, benefits were seen for total nasopharyngeal area, upper/lower airway dimensions and upper/lower pharynx dimensions (Table 3). However, most of these changes were small to moderate in magnitude, which means that they might have little clinical relevance (Supplementary Materials S7-S10). The only exception was the increase in total nasopharyngeal area, where a large to very large effect was (Supplementary Materials S6) found. This indicates that any clinically relevant benefits in airway dimensions or breathing might be located in this compartment. Evidence from the existing literature indicates that significant differences exist in the dimensions of the pharyngeal airway and the thickness of the pharyngeal wall among normal patients and patients with sleep-disordered breathing [35], with the lower retropalatal and retroglossal areas being affected the most [36]. Even though these regions were only minimally affected, the large improvements at the nasopharyngeal area might be highly relevant, since the nasopharyngeal dimensions appear to be the most sensitive parameter for assessments of the patient's respiratory conditions [37]. However, even though dimensional changes might be indicative of improved breathing, proper confirmation must follow using functional analyses of nasal airflow resistance and nasal pressure.

The initial effects on the airways observed after treatment were, for the most part, retained after a follow-up period of 2-5 years, while in some instances, the difference between treated and untreated Class III patients even increased (Table 3). This might indicate that early orthopedic modification of a maxillary retrusion might be associated with a more favorable growth pattern, even though this is speculative at the present time. A long-term follow-up from a well-known randomized trial on maxillary protraction indicated relatively similar change patterns for SNA, SNB and ANB angles from 8 to 14 years of age for treated and untreated Class III patients, with mandibular rotation being the most pronounced difference [38]. Different extents of mandibular rotation, as expressed through the patient's growth pattern, were observed to be differently related to airway dimensions in Class I patients [39]. Apart from that, therapeutic effects should always be considered in the context of physiological growth. The dimensions of the nasopharyngeal compartment are not stable during growth [40,41], and patient age at the time of the investigation could play a role in the interpretation of the results.

The effects of orthopedic maxillary traction on the airways were relatively consistent, despite the big variability of the included studies regarding patient characteristics (age, sex, baseline airway dimensions) and treatment protocol (simultaneous use of maxillary expansion or treatment duration), as no clear modification was seen through the subgroup/meta-regression analyses (Table 4). Previous studies have indicated that maxillary expansion can have a beneficial effect on upper airway dimensions [42], but whether additional gains can be expected during maxillary protraction by also incorporating expansion remains questionable. It must be also noted here that alternating rapid maxillary expansion and constriction might be more beneficial than conventional rapid maxillary expansion in terms of skeletal effects [43], but no included studies used this protocol. 
Restricting or redirecting mandibular growth with a chin-cup had a considerably smaller impact on the airways than maxillary protraction, since only two statistically significant differences directly post-treatment were found, and only one was clinically relevant (the increase in nasopharyngeal area) (Supplementary Materials S11). However, it must be noted that only two small studies were included in this review, which might indicate an absence of evidence, and not necessarily evidence of absence. Chin-cup treatment has been reported to induce certain skeletal adaptations [44,45], the long-term stability of which, however, is questionable. At the present time, there is not enough evidence to suggest that chin-cup treatment negatively influences airway dimensions.

\subsection{Strengths and Limitations}

This systematic review has several strengths, comprising an a priori registered protocol [14], a comprehensive literature search, the inclusion of an untreated Class III control group, the use of modern analytic methods [21], the application of the GRADE approach to assess the strength of provided recommendations [17], and the transparent provision of all data [46].

Some limitations exist nonetheless. For one, methodological issues, which might influence the present conclusions, existed in all the included studies. This is especially the case in the included retrospective nonrandomized studies $[47,48]$. The inclusion of nonrandomized studies in meta-analyses is not considered prohibitory per se, provided that robust bias appraisal has been performed and recent guidance has been provided on how to appropriately incorporate such designs [24]. Furthermore, most meta-analyses were predominantly based on small trials, which might affect the precision of the estimates [49]. Additionally, the small number of trials ultimately included in the meta-analyses and their incomplete reporting of results and potential confounders (baseline malocclusion severity or other patient characteristics and different orthopedic treatment protocols) prevented us from conducting many analyses for subgroups and meta-regressions. Last but not least, this study, although it was originally otherwise planned, only included lateral cephalograms for evaluating airway dimensions because no eligible controlled study with CBCTs could be found. Lateral cephalograms are abundantly available because they are part of patients' usual records, but only a moderately high correlation is to be expected between cephalometric and CBCT measurements in the assessment of the airway dimensions [50].

\section{Conclusions}

Current evidence indicates that orthopedic treatment with maxillary protraction for Class III malocclusion might be associated with increased dimensions of the upper airways, which seem to be retained after treatment. However, our confidence in these data is very low due to the poor quality of existing studies and their small number. Restriction of mandibular growth with chin-cup seems likewise to be associated to some extent with increased airway dimensions, but these effects are less pronounced. It is crucial that the clinical relevance of such anatomical changes be confirmed by functional analyses of breathing ability before concrete recommendations can be formulated.

Supplementary Materials: The following are available online at http://www.mdpi.com/2077-0383/9/9/ 3015/s1, Supplementary Materials S1: Additional review details and deviations from the protocol, Supplementary Materials S2: Eligibility criteria for the inclusion of primary studies, Supplementary Materials S3: Literature search (as of 4 August 2020) for each database with the corresponding hits, Supplementary Materials S4: List of studies identified from the literature search and their inclusion/exclusion status with reasons, Supplementary Materials S5: Results of individual studies comparing maxillary protraction to untreated controls that are not included in meta-analyses, Supplementary Materials S6: Contour-enhanced forest plot for the comparison of maxillary protraction with facemask versus changes in untreated controls in terms of total nasopharyngeal area, Supplementary Materials S7: Contour-enhanced forest plot for the comparison of maxillary protraction with facemask versus changes in untreated controls in terms of upper airway dimension, Supplementary Materials S8: Contour-enhanced forest plot for the comparison of maxillary protraction with facemask versus changes in untreated controls in terms of lower airway dimension, Supplementary Materials S9: Contour-enhanced forest plot for the comparison of maxillary protraction with facemask versus changes in untreated controls in terms of McNamara's upper pharynx dimension, Supplementary Materials S10: Contour-enhanced forest plot for the comparison of maxillary protraction with facemask versus changes 
in untreated controls in terms of McNamara's lower pharynx dimension, Supplementary Materials S11: Results of individual studies comparing chin-cup to untreated controls that are not included in meta-analyses, Supplementary Materials S12: Sensitivity analyses of meta-analyses post-treatment with at least 3 studies.

Author Contributions: G.H. wrote the first draft of the protocol, while V.K., T.E., and S.N.P. revised it. G.H. and S.N.P. did the literature searches. G.H. and V.K. independently performed study selection in duplicate, data extraction, and risk of bias assessment. Disagreements were resolved with two authors S.N.P. and T.E. Literature searches and data analysis were performed by the last author (S.N.P.). G.H. wrote the first draft of the manuscript, while V.K., T.E., and S.N.P. revised it. All authors have read and agreed to the published version of the manuscript.

Funding: This research received no external funding.

Conflicts of Interest: The authors declare no conflict of interest.

\section{References}

1. Jayaratne, Y.S.N.; Zwahlen, R.A. The oropharyngeal airway in young adults with skeletal Class II and Class III deformities: A 3-D morphometric analysis. PLoS ONE 2016, 11, e0148086. [CrossRef] [PubMed]

2. El, H.; Palomo, J.M. Airway volume for different dentofacial skeletal patterns. Am. J. Orthod. Dentofac. Orthop. 2011, 139, e511-e521. [CrossRef] [PubMed]

3. Iwasaki, T.; Suga, H.; Yanagisawa-Minami, A.; Sato, H.; Sato-Hashiguchi, M.; Shirazawa, Y.; Tsujii, T.; Yamamoto, Y.; Kanomi, R.; Yamasaki, Y. Relationships among tongue volume, hyoid position, airway volume and maxillofacial form in paediatric patients with Class-I, Class-II and Class-III malocclusions. Orthod. Craniofacial Res. 2018, 22, 9-15. [CrossRef] [PubMed]

4. Pacheco, M.C.T.; Fiorott, B.S.; Finck, N.S.; De Araújo, M.T.M. Craniofacial changes and symptoms of sleep-disordered breathing in healthy children. Dent. Press J. Orthod. 2015, 20, 80-87. [CrossRef]

5. Dantas, J.; Neto, J.; De Carvalho, S.; Martins, I.; De Souza, R.; Sarmento, V.A. Satisfaction of skeletal class III patients treated with different types of orthognathic surgery. Int. J. Oral Maxillofac. Surg. 2015, 44, $195-202$. [CrossRef]

6. Havron, A.G.; Aronovich, S.; Shelgikar, A.V.; Kim, H.L.; Conley, R.S. 3D airway changes using CBCT in patients following mandibular setback surgery \pm maxillary advancement. Orthod. Craniofacial Res. 2019, 22, 30-35. [CrossRef]

7. Hiyama, S.; Suda, N.; Ishii-Suzuki, M.; Tsuiki, S.; Ogawa, M.; Suzuki, S.; Kuroda, T. Effects of maxillary protraction on craniofacial structures and upper-airway dimension. Angle Orthod. 2002, 72, 43-47.

8. Lanteri, V.; Farronato, M.; Ugolini, A.; Cossellu, G.; Gaffuri, F.; Parisi, F.M.R.; Cavagnetto, D.; Abate, A.; Maspero, C. Volumetric changes in the upper airways after rapid and slow maxillary expansion in Growing patients: A case-control study. Materials 2020, 13, 2239. [CrossRef]

9. Tuncer, B.B.; Kaygisiz, E.; Tuncer, C.; Yüksel, S. Pharyngeal airway dimensions after chin cup treatment in Class III malocclusion subjects. J. Oral Rehabil. 2009, 36, 110-117. [CrossRef]

10. Maspero, C.; Farronato, M.; Bellincioni, F.; Annibale, A.; Machetti, J.; Abate, A.; Cavagnetto, D.; Maspero, C. Three-dimensional evaluation of maxillary sinus changes in growing subjects: A retrospective cross-sectional study. Materials 2020, 13, 1007. [CrossRef]

11. Mucedero, M.; Baccetti, T.; Franchi, L.; Cozza, P. Effects of maxillary protraction with or without expansion on the sagittal pharyngeal dimensions in Class III subjects. Am. J. Orthod. Dentofac. Orthop. 2009, 135, 777-781. [CrossRef] [PubMed]

12. Baccetti, T.; Franchi, L.; Mucedero, M.; Cozza, P. Treatment and post-treatment effects of facemask therapy on the sagittal pharyngeal dimensions in Class III subjects. Eur. J. Orthod. 2010, 32, 346-350. [CrossRef] [PubMed]

13. Ming, Y.; Hu, Y.; Li, Y.; Yu, J.; He, H.; Zheng, L. Effects of maxillary protraction appliances on airway dimensions in growing class III maxillary retrognathic patients: A systematic review and meta-analysis. Int. J. Pediatr. Otorhinolaryngol. 2018, 105, 138-145. [CrossRef]

14. Sideri, S.; Papageorgiou, S.N.; Eliades, T. Registration in the international prospective register of systematic reviews (PROSPERO) of systematic review protocols was associated with increased review quality. J. Clin. Epidemiol. 2018, 100, 103-110. [CrossRef] [PubMed] 
15. Sterne, J.A.C.; Hernán, M.A.; Reeves, B.C.; Savović, J.; Berkman, N.D.; Viswanathan, M.; Henry, D.; Altman, D.G.; Ansari, M.T.; Boutron, I.; et al. ROBINS-I: A tool for assessing risk of bias in non-randomised studies of interventions. BMJ 2016, 355, i4919. [CrossRef]

16. Papageorgiou, S.N. Meta-analysis for orthodontists: Part II-Is all that glitters gold? J. Orthod. 2014, 41, 327-336. [CrossRef]

17. Guyatt, G.H.; Oxman, A.D.; Schünemann, H.; Tugwell, P.; Knottnerus, A. GRADE guidelines: A new series of articles in the Journal of Clinical Epidemiology. J. Clin. Epidemiol. 2011, 64, 380-382. [CrossRef]

18. Higgins, J.P.T.; Green, S. Cochrane Handbook for Systematic Reviews of Interventions Version 5.1.0; The Cochrane Collaboration: London, UK, 2011.

19. Liberati, A.; Altman, D.G.; Tetzlaff, J.; Mulrow, C.; Gøtzsche, P.C.; Ioannidis, J.P.; Clarke, M.J.; Devereaux, P.J.; Kleijnen, J.; Moher, D. The PRISMA statement for reporting systematic reviews and meta-analyses of studies that evaluate health care interventions: Explanation and elaboration. J. Clin. Epidemiol. 2009, 62, e1-e34. [CrossRef]

20. Papageorgiou, S.N. Meta-analysis for orthodontists: Part I-How to choose effect measure and statistical model. J. Orthod. 2014, 41, 317-326. [CrossRef]

21. Langan, D.; Higgins, J.P.T.; Jackson, D.; Bowden, J.; Veroniki, A.A.; Kontopantelis, E.; Viechtbauer, W.; Simmonds, M. A comparison of heterogeneity variance estimators in simulated random-effects meta-analyses. Res. Synth. Methods 2018, 10, 83-98. [CrossRef]

22. Higgins, J.P.T.; Thompson, S.G.; Deeks, J.J.; Altman, D.G. Measuring inconsistency in meta-analyses. BMJ 2003, 327, 557-560. [CrossRef] [PubMed]

23. IntHout, J.; Ioannidis, J.P.A.; Rovers, M.M.; Goeman, J.J. Plea for routinely presenting prediction intervals in meta-analysis. BMJ Open 2016, 6. [CrossRef] [PubMed]

24. Schünemann, H.J.; Cuello-Garcia, C.A.; A Akl, E.; A Mustafa, R.; Meerpohl, J.J.; Thayer, K.A.; Morgan, R.L.; Gartlehner, G.; Kunz, R.; Katikireddi, S.V.; et al. GRADE guidelines: 18. How ROBINS-I and other tools to assess risk of bias in nonrandomized studies should be used to rate the certainty of a body of evidence. J. Clin. Epidemiol. 2018, 111, 105-114. [CrossRef] [PubMed]

25. Carrasco-Labra, A.; Brignardello-Petersen, R.; Santesso, N.; Neumann, I.; A Mustafa, R.; Mbuagbaw, L.; Etxeandia-Ikobaltzeta, I.; De Stio, C.; Mccullagh, L.J.; Alonso-Coello, P.; et al. Improving GRADE evidence tables part 1: A randomized trial shows improved understanding of content in summary of findings tables with a new format. J. Clin. Epidemiol. 2016, 74, 7-18. [CrossRef] [PubMed]

26. Havakeshian, G.; Koretsi, V.; Eliades, T.; Papageorgiou, S.N. Effect of Orthopedic Treatment for Class III Malocclusion on Upper Airways: A Systematic Review and Meta-Analysis [Dataset]. Available online: https://zenodo.org/record/3988960 (accessed on 18 August 2020). [CrossRef]

27. Ioannidis, J.P.A. Interpretation of tests of heterogeneity and bias in meta-analysis. J. Eval. Clin. Pract. 2008, 14, 951-957. [CrossRef] [PubMed]

28. Akin, M.; Ucar, F.; Chousein, C.; Sari, Z. Effects of chincup or facemask therapies on the orofacial airway and hyoid position in Class III subjects. J. Orofac. Orthop. 2015, 76, 520-530. [CrossRef]

29. Tuncer, B.B.; Ulusoy, Ç.; Tuncer, C.; Türköz, Ç.; Varlik, S.K. Effects of reverse headgear on pharyngeal airway in patients with different vertical craniofacial features. Braz. Oral Res. 2015, 29, 1-8. [CrossRef]

30. Danaei, S.M.; Ajami, S.; Etemadi, H.; Azadeh, N. Assessment of the effect of maxillary protraction appliance on pharyngeal airway dimensions in relation to changes in tongue posture. Dent. Res. J. 2018, 15, 208-214. [CrossRef]

31. Kilinc, A.S.; Arslan, S.G.; Kama, J.D.; Ozer, T.; Dari, O. Effects on the sagittal pharyngeal dimensions of protraction and rapid palatal expansion in Class III malocclusion subjects. Eur. J. Orthod. 2008, 30, 61-66. [CrossRef]

32. Lombardo, E.; Franchi, L.; Lione, R.; Chiavaroli, A.; Cozza, P.; Pavoni, C. Evaluation of sagittal airway dimensions after face mask therapy with rapid maxillary expansion in Class III growing patients. Int. J. Pediatr. Otorhinolaryngol. 2020, 130, 109794. [CrossRef]

33. Menendez-Diaz, I.; Muriel, J.; Cobo, J.L.; Alvarez, C.; Cobo, T. Early treatment of Class III malocclusion with facemask therapy. Clin. Exp. Dent. Res. 2018, 4, 279-283. [CrossRef] [PubMed]

34. Yagci, A.; Uysal, T.; Usumez, S.; Orhan, M. Effects of modified and conventional facemask therapies with expansion on dynamic measurement of natural head position in Class III patients. Am. J. Orthod. Dentofac. Orthop. 2011, 140, e223-e231. [CrossRef] [PubMed] 
35. Schwab, R.J.; Gupta, K.B.; Gefter, W.B.; Metzger, L.J.; Hoffman, E.A.; Pack, A.I. Upper airway and soft tissue anatomy in normal subjects and patients with sleep-disordered breathing. Significance of the lateral pharyngeal walls. Am. J. Respir. Crit. Care Med. 1995, 152, 1673-1689. [CrossRef] [PubMed]

36. Schwab, R.J.; Gefter, W.B.; Hoffman, E.A.; Gupta, K.B.; Pack, A.I. Dynamic upper airway imaging during awake respiration in normal subjects and patients with sleep disordered breathing. Am. Rev. Respir. Dis. 1993, 148, 1385-1400. [CrossRef]

37. Poole, M.N.; Engel, G.A.; Chaconas, S.J. Nasopharyngeal cephalometrics. Oral Surg. Oral Med. Oral Pathol. 1980, 49, 266-271. [CrossRef]

38. Mandall, N.; Cousley, R.; DiBiase, A.; Dyer, F.; Littlewood, S.; Mattick, R.; Nute, S.J.; Doherty, B.; Stivaros, N.; McDowall, R.; et al. Early class III protraction facemask treatment reduces the need for orthognathic surgery: A multi-centre, two-arm parallel randomized, controlled trial. J. Orthod. 2016, 43, 164-175. [CrossRef]

39. Ucar, F.I.; Uysal, T. Orofacial airway dimensions in subjects with Class I malocclusion and different growth patterns. Angle Orthod. 2011, 81, 460-468. [CrossRef]

40. Linder-Aronson, S.; Leighton, B.C. A longitudinal study of the development of the posterior nasopharyngeal wall between 3 and 16 years of age. Eur. J. Orthod. 1983, 5, 47-58. [CrossRef]

41. Preston, C.; Tobias, P.V.; Salem, O.H. Skeletal age and growth of the nasopharynx in the sagittal plane: A cephalometric study. Semin. Orthod. 2004, 10, 16-38. [CrossRef]

42. Buck, L.M.; Dalci, O.; Darendeliler, M.A.; Papageorgiou, S.N.; Papadopoulou, A.K. Volumetric upper airway changes after rapid maxillary expansion: A systematic review and meta-analysis. Eur. J. Orthod. 2016, 39, 463-473. [CrossRef]

43. Almuzian, M.; McConnell, E.; Darendeliler, M.A.; Alharbi, F.; Mohammed, H. The effectiveness of alternating rapid maxillary expansion and constriction combined with maxillary protraction in the treatment of patients with a class III malocclusion: A systematic review and meta-analysis. J. Orthod. 2018, 45, 250-259. [CrossRef] [PubMed]

44. Zurfluh, M.A.; Kloukos, D.; Patcas, R.; Eliades, T. Effect of chin-cup treatment on the temporomandibular joint: A systematic review. Eur. J. Orthod. 2014, 37, 314-324. [CrossRef] [PubMed]

45. Rongo, R.; D’Antò, V.; Bucci, R.; Polito, I.; Martina, R.; Michelotti, A. Skeletal and dental effects of Class III orthopaedic treatment: A systematic review and meta-analysis. J. Oral Rehabil. 2017, 44, 545-562. [CrossRef] [PubMed]

46. Papageorgiou, S.N.; Cobourne, M.T. Data sharing in orthodontic research. J. Orthod. 2018, 45, 1-3. [CrossRef]

47. Papageorgiou, S.N.; Xavier, G.M.; Cobourne, M.T. Basic study design influences the results of orthodontic clinical investigations. J. Clin. Epidemiol. 2015, 68, 1512-1522. [CrossRef]

48. Papageorgiou, S.N.; Koretsi, V.; Jäger, A. Bias from historical control groups used in orthodontic research: A meta-epidemiological study. Eur. J. Orthod. 2016, 39, 98-105. [CrossRef]

49. Cappelleri, J.C.; Loannidis, J.P.A.; Schmid, C.H.; De Ferranti, S.D.; Aubert, M.; Chalmers, T.C.; Lau, J. Large Trials vs. Meta-analysis of Smaller Trials. JAMA 1996, 276, 1332-1338. [CrossRef]

50. Aboudara, C.; Nielsen, I.; Huang, J.C.; Maki, K.; Miller, A.J.; Hatcher, D. Comparison of airway space with conventional lateral headfilms and 3-dimensional reconstruction from cone-beam computed tomography. Am. J. Orthod. Dentofac. Orthop. 2009, 135, 468-479. [CrossRef]

(C) 2020 by the authors. Licensee MDPI, Basel, Switzerland. This article is an open access article distributed under the terms and conditions of the Creative Commons Attribution (CC BY) license (http://creativecommons.org/licenses/by/4.0/). 